\title{
Die FMH begrüsst das neue Psychologieberufe-Gesetz
}

\section{Christine Romann}

Mitglied des Zentralvorstands der FMH, Verantwortliche Ressort Gesundheitsförderung und Prävention
Korrespondenz:

Dr. med. Christine Romann

FMH

Elfenstrasse 18

CH-3000 Bern 15

Tel. 0313591111

Fax 0313591112

christine.romann@bluewin.ch
Wer die Dienste einer Psychologin, eines Psychologen in Anspruch nimmt, möchte sicher sein, dass er oder sie sich einer gutausgebildeten Person anvertraut. Dies gilt insbesondere im heiklen Bereich der psychologischen Psychotherapie, die - wie die Psychologieberufe insgesamt - schon seit längerem einer gesetzlichen Regelung harrt. Bereits 1991 ersuchte die Schweizerische Konferenz der Gesundheitsdirektoren und -direktorinnen den Bundesrat um die Ausarbeitung einer Bundesregelung für die Aus-, Weiter- und Fortbildung der Psychotherapeuten - damals noch innerhalb der Medizinalberufe. Das wurde vielerorts heftig kritisiert, und 1998 erhielt das Eidgenössische Departement des Innern vom Bundesrat den Auftrag, ein Bundesgesetz im Bereich der Psychologie auszuarbeiten. 2001 erhielt dieses Vorhaben auch vom Parlament Unterstützung, das entsprechende parlamentarische Vorstösse deutlich annahm. So ging schliesslich 2005 ein erster Vorentwurf zum jetzigen Gesetz in die Vernehmlassung, wo er allerdings wieder viel Kritik erntete. Nach einer gründlichen Überarbeitung legte man den Gesetzesentwurf im Februar 2009 nochmals einem interessierten Kreis in einem Hearing vor. Das so mehrfach überarbeitete Gesetz geht nun seiner Beratung in den eidgenössischen Räten entgegen.

Zielsetzung des Psychologieberufe-Gesetzes ist der Schutz der psychischen Gesundheit: Menschen, die psychologische Dienstleistungen in Anspruch nehmen, sollen vor Täuschung durch die behandelnde Psychologin oder den behandelnden Psychologen geschützt werden. Mit klaren, geschützten Berufsbezeich- nungen und mit eidgenössischen Weiterbildungstiteln will der Bundesrat dies gewährleisten. Das Gesetz sieht vor, dass nur noch Personen mit einem Master in Psychologie berechtigt sind, sich Psychologin bzw. Psychologe zu nennen und unter dieser Bezeichnung ihre Dienstleistungen anzubieten. Der Bereich der psychologischen Psychotherapie wird weitergehend geregelt: Voraussetzung für die Weiterbildung zum Psychotherapeuten oder zur Psychotherapeutin ist ein Master in Psychologie - wobei das Fach «Klinische Psychologie» im Ausbildungsgang eingeschlossen sein muss. Zudem müssen die Weiterbildungsgänge zur psychologischen Psychotherapie eidgenössisch akkreditiert sein. Eine gewisse Durchlässigkeit sieht der Gesetzesvorschlag in der universitären Ausbildung vor: Inhaberinnen und Inhaber psychologienaher Hochschulabschlüsse sollen zu angemessenen Bedingungen den Zugang zur Ausbildung in Psychologie haben. Die FMH befürwortet eine weitergehende Lösung: Wer einen Bachelor in einem psychologienahen Studium hat, beispielsweise Pädagogik, erhält Zugang zum Masterstudium der Psychologie.

Der Gesetzesentwurf vermag insgesamt zu überzeugen und wird seinem Grundanliegen gerecht. Der Titelschutz schafft Klarheit für Konsumentinnen und Konsumenten. Mit der Einführung der akkreditierten Weiterbildung zur psychologischen Psychotherapeutin bzw. zum psychologischen Psychotherapeuten wird die in diesem sensiblen Bereich notwendige hohe Qualität der psychologischen Psychotherapie angestrebt.

\section{Ärztekammer - Wahl von zwei Mitgliedern für den FMH-Zentralvorstand}

Zwei bisherige Mitglieder des Zentralvorstandes, Dr. med. Marie-Christine Peter und Dr. med. Max Giger, treten nach langjähriger erfolgreicher Tätigkeit per 27. Mai 2010 zurück.

An der ordentlichen Ärztekammer vom 27. Mai 2010 steht darum die Nachfolge bzw. die Wahl von zwei neuen Zentralvorstandsmitgliedern für die freiwerdenden Sitze an.

Wir möchten den Kandidatinnen und Kandidaten die Möglichkeit geben, sich zuvor in der Schwei- zerischen Ärztezeitung vorzustellen. Für entsprechende Informationen kontaktieren Sie uns bitte über kommunikation@fmh.ch. Gerne stellen wir Ihnen auf Anfrage ein Dokument mit den benötigten Angaben sowie Beispiele solcher Porträts zu. Ihre Angaben benötigen wir bis spätestens 19. April 2010. Die Porträts erscheinen in der Schweizerischen Ärztezeitung vom 12. Mai 2010.

Kommunikation FMH 